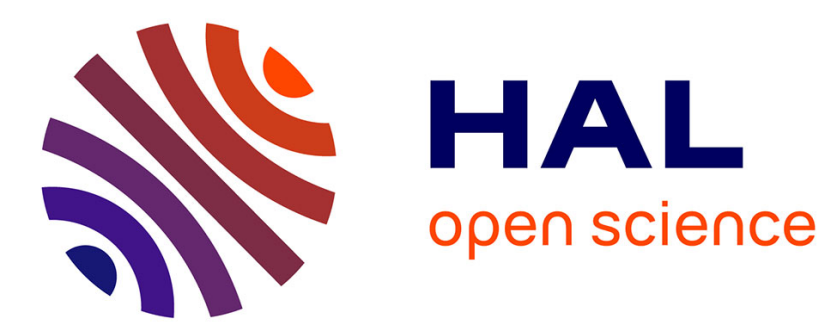

\title{
Shallow and deep levels in carbon-doped hexagonal boron nitride crystals
}

Thomas Pelini, Christine Elias, R. Page, L. Xue, S. Liu, J. Li, J. H Edgar, Anaïs Dréau, Vincent Jacques, Pierre Valvin, et al.

\section{- To cite this version:}

Thomas Pelini, Christine Elias, R. Page, L. Xue, S. Liu, et al.. Shallow and deep levels in carbon-doped hexagonal boron nitride crystals. Physical Review Materials, 2019, 3, pp.094001. 10.1103/PhysRevMaterials.3.094001 . hal-02289672

\section{HAL Id: hal-02289672 \\ https://hal.science/hal-02289672}

Submitted on 11 Jan 2021

HAL is a multi-disciplinary open access archive for the deposit and dissemination of scientific research documents, whether they are published or not. The documents may come from teaching and research institutions in France or abroad, or from public or private research centers.
L'archive ouverte pluridisciplinaire $\mathbf{H A L}$, est destinée au dépôt et à la diffusion de documents scientifiques de niveau recherche, publiés ou non, émanant des établissements d'enseignement et de recherche français ou étrangers, des laboratoires publics ou privés. 


\title{
Shallow and deep levels in carbon-doped hexagonal boron nitride crystals
}

\author{
T. Pelini, ${ }^{1}$ C. Elias, ${ }^{1}$ R. Page,${ }^{2}$ L. Xue,${ }^{3}$ S. Liu,${ }^{3}$ J. Li, ${ }^{3}$, J. H. \\ Edgar, ${ }^{3}$ A. Dréau, ${ }^{1}$ V. Jacques, ${ }^{1}$ P. Valvin, ${ }^{1}$ B. Gil,${ }^{1}$ G. Cassabois ${ }^{1, *}$ \\ ${ }^{1}$ Laboratoire Charles Coulomb UMR 5221 CNRS-Université de Montpellier, 34095 Montpellier, France \\ ${ }^{2}$ Department of Materials Science and Engineering, \\ Cornell University, Ithaca, New York 14853, USA \\ 3 Tim Taylor Department of Chemical Engineering, \\ Kansas State University, Manhattan Kansas 66506, USA
}

(Dated: August 21, 2019)

\begin{abstract}
We study shallow and deep levels in carbon-doped hexagonal boron nitride crystals precipitated from a molten metal solution in a high temperature furnace. Reflectance and photoluminescence under deep ultraviolet excitation are complemented by spatially-resolved experiments by means of a scanning confocal micro-photoluminescence setup operating in the ultraviolet. Isotopicallycontrolled carbon doping does not induce any energy shift of the well-known deep level emission at 4.1 $\mathrm{eV}$. Our detailed characterization in a series of carbon-doped crystals reveals that the incorporation of carbon during the growth process results in a distinct class of shallow and deep levels in hexagonal boron nitride, calling into question the exact role of carbon in the growth of hexagonal boron nitride and its direct or indirect influence on the formation of the crystal defects.
\end{abstract}

\section{INTRODUCTION}

Hexagonal boron nitride $(\mathrm{hBN})$ is a semiconducting material which has gathered increasing attention due to its unique properties. As a lamellar compound, hBN is a model 2D material along with graphene and transition metal dichalcogenides, and a fundamental building block of van der Waals heterostructures [1]. As a wide-band gap nitride semiconductor, hBN possesses great potential for deep ultraviolet (UV) applications due to the bright luminescent emission of hBN single crystals [2], thus competing with nitride semiconductors of the AlGaN family that are already widely used for blue and UV lighting [3]. The scalable and controlled growth of high-quality hBN, however, is the current challenge precluding future developments in many communities that share a common interest for this outstanding material [4].

A key issue to understanding the growth of a crystal is to understand the nature of its defects. On one hand, the suppression of extended and point defects is a prerequisite for the fabrication of large hBN single crystals. Although recent studies have reported significant progress in the limit of thin samples with the growth of monolayer $\mathrm{hBN}$ by chemical vapor deposition $[5,6]$ and molecular beam epitaxy [7], the synthesis of thick epilayers does not lead yet to a crystalline quality competing with bulk hBN crystals grown by precipitation techniques $[2,8,9]$. On the other hand, the controlled creation of defects is important in the context of quantum technologies where point defects are used as artifical atoms in solid state [10], and also in the traditional prospect of doping for implementing electrical injection in commercial devices.

Carbon was early proposed as an impurity playing an important role in the opto-electronic properties of $\mathrm{hBN}$ $[11,12]$. After the growth of high-purity hBN crystals in 2004 [2], Taniguchi et al. reported a strong increase in the photoluminescence (PL) intensity of the $4.1 \mathrm{eV}$ line in hBN crystals with a high carbon concentration [13]. Still, there is no consensus on the exact role of carbon in the physics of the defects in $\mathrm{hBN}$, with a lively debate on both experimental [14-17] and theoretical [18-21] sides.

Here, we have studied the optical properties of carbondoped hBN. We have performed reflectance and PL under deep UV excitation, complemented by spatially-resolved experiments by means of a home-made UV scanning confocal micro-PL setup on a series of hBN crystals grown by precipitation from a molten metal solution in a high temperature furnace. In the spectral range of shallow levels extending from 5 to $5.7 \mathrm{eV}$, we identify new optical transitions at 5.37 and $5.52 \mathrm{eV}$ that we attribute to defect bound excitons. In the UV range of deep levels emitting around $4 \mathrm{eV}$, we first focus on the well-known $4.1 \mathrm{eV}$ line and examine if isotopically-controlled doping by ${ }^{13} \mathrm{C}$ modifies its emission energy. Then, we report the observation of additional optical transitions observed from 4.12 to $4.16 \mathrm{eV}$, which remain unaddressed in the literature. We combine reflectance, temperature-dependent $\mathrm{PL}$, and spatially-resolved micro-PL to demonstrate they correspond to a class of point defects, distinct from the $4.1 \mathrm{eV}$ line.

This paper is organized as follows. Section II is devoted to the presentation of our experiments, with a description of the optical measurements (Section II A) and of our hBN crystals (Section IIB). We then present our experimental results on the shallow levels (Section III) and on the deep levels (Section IV) in our carbon-doped hBN crystals. 


\begin{tabular}{|c|c|c|c|c|c|}
\hline Sample name & Graphite quantity & BN quantity & Solvents & Cooling rate & Atmosphere \\
\hline & & & & & \\
$\mathrm{C} 20$ & $1.5 \mathrm{~g}$ graphite powder & $0.5 \mathrm{~g} \mathrm{BN}$ powder & $10 \mathrm{~g} \mathrm{Fe}+10 \mathrm{~g} \mathrm{Cr}$ & $4^{\circ} \mathrm{C} / \mathrm{hour}$ & $\mathrm{H}_{2}+\mathrm{N}_{2}$ \\
$\mathrm{C} 22$ & $1.5 \mathrm{~g}$ graphite powder & $0.5 \mathrm{~g} \mathrm{BN}$ powder & $10 \mathrm{~g} \mathrm{Ni}+10 \mathrm{~g} \mathrm{Cr}$ & $4{ }^{\circ} \mathrm{C} / \mathrm{hour}$ & $\mathrm{H}_{2}+\mathrm{N}_{2}$ \\
$\mathrm{C} 27$ & graphite piece & $\mathrm{HPBN}$ & $17 \mathrm{~g} \mathrm{Fe}+17 \mathrm{~g} \mathrm{Cr}$ & $4^{\circ} \mathrm{C} / \mathrm{hour}$ & $\mathrm{H}_{2}+\mathrm{N}_{2}$ \\
$\mathrm{C} 33$ & $2.0 \mathrm{~g}$ graphite powder & $1.0 \mathrm{~g} \mathrm{BN}$ powder & $20 \mathrm{~g} \mathrm{Ni}+20 \mathrm{~g} \mathrm{Cr}$ & $0.5^{\circ} \mathrm{C} / \mathrm{hour}$ & $\mathrm{CO}+\mathrm{N}_{2}$ \\
$\mathrm{C} 40$ & $0.75 \mathrm{~g}{ }^{13} \mathrm{C}$ powder & $\mathrm{BN}$ pressed boat & $10 \mathrm{~g} \mathrm{Ni}+10 \mathrm{~g} \mathrm{Cr}$ & $0.5^{\circ} \mathrm{C} /$ hour & $\mathrm{CO}+\mathrm{N}_{2}$ \\
\hline
\end{tabular}

TABLE I. Growth parameters of the carbon-doped hBN crystals precipitated from a molten metal solution in a high temperature furnace. HPBN stands for Hot Pressed Boron Nitride.

\section{EXPERIMENTS}

In this section, we present our experiments dealing with optical spectroscopy by reflectivity, macro- and micro-PL (Section II A), performed in various hBN crystals for which we detail the synthesis protocol and conditions, complemented by basic elementary characterization by PL over a large spectral range from 3.5 to $6 \mathrm{eV}$ (Section II B).

\section{A. Optics}

\section{Reflectivity}

A home-made reflectance setup was built based on a deuterium lamp (63163 Newport), spectrally filtered through a monochromator (Cornerstone CS130) with 100 $\mu \mathrm{m}$-slits providing a spectral resolution on the order of 10 $\mathrm{meV}$. The incident light was focused on the sample close to normal incidence, with a rectangular spot of size 100 $\mu \mathrm{m} \times 1.5 \mathrm{~mm}$. All optical elements are reflective mirrors coated for deep UV. For more details on the reflectance setup, please refer to Ref.[7].

\section{Macro-photoluminescence}

In macro-PL spectroscopy, we performed above bandgap excitation at $195 \mathrm{~nm}(6.35 \mathrm{eV})$ with the fourth harmonic of a $\mathrm{cw}$ mode-locked Ti:Sa oscillator with a repetition rate of $82 \mathrm{MHz}$. The spot diameter is on the order of $50 \mu \mathrm{m}$, with a power of $30 \mu \mathrm{W}$. An achromatic optical system couples the emitted signal to our detection system, composed of a $\mathrm{f}=300 \mathrm{~mm}$ Czerny-Turner monochromator, equipped with a 1800 grooves $/ \mathrm{mm}$ grating blazed at $250 \mathrm{~nm}$ (providing a spectral resolution of $0.8 \mathrm{~nm}$ or $2.5 \mathrm{meV}$ at $200 \mathrm{~nm}$ ), and with a back-illuminated CCD camera (Andor Newton 920), with a quantum efficiency of $50 \%$ at $210 \mathrm{~nm}$, operated over integration times of 5 min. The sample is held on the cold finger of a closedcycle cryostat for temperature-dependent measurements from $10 \mathrm{~K}$ to room temperature.

\section{Micro-photoluminescence}

For micro-PL spectroscopy, the excitation is below the band-gap of hBN with a diode-pumped cw laser providing an optical emission at $266 \mathrm{~nm}(4.66 \mathrm{eV})$ coming from the fourth harmonic of a Nd:YAG laser. The setup is a home-made confocal microscope based on a closedcycle cryostat with a high numerical aperture objective $(\mathrm{NA}=0.4)$ optimized for UV spectroscopy. Our lateral spatial resolution is about $700 \mathrm{~nm}$ and the longitudinal one of about $1 \mu \mathrm{m}$. The PL signal is detected with an achromatic and geometric aberration-free optical system and coupled, either to hybrid photomultiplier tubes (PMA Series PicoQuant) connected to a single-photon counting acquisition card, or to a spectrometer (Shamrock 500i, f=500 mm Czerny-Turner system) equipped with a grating of 1800 grooves $/ \mathrm{mm}$ blazed at $250 \mathrm{~nm}$ (providing a spectral resolution of $0.02 \mathrm{~nm}$ or $0.3 \mathrm{meV}$ at $300 \mathrm{~nm}$ ) and a back-illuminated UV-enhanced camera (Andor Newton DU940P BU2) with a quantum efficiency of $60 \%$ at $300 \mathrm{~nm}$.

\section{B. Samples}

\section{Synthesis of hBN crystals}

The carbon-doped hBN single crystals were grown from a molten metal solution at atmospheric pressure with a modified version of the growth procedure described in Ref.[8, 9]. Three metals were used: nickel, iron, and chromium. Nickel and iron are good solvents for boron, with maximum solubilities of 18.5 at $\%$ and 17 at\%, respectively. Chromium is good for dissolving nitrogen. Mixing of both metallic solvents forms an eutectic (at $1345^{\circ} \mathrm{C}$ for $\mathrm{Ni}$ and $\mathrm{Cr}$ ). The boron source for all samples were either a commercial hBN powder or Hot Pressed Boron Nitride (HPBN), nominally fabricated from the same commercial powder. The carbon source was graphite powder, with the natural distribution of carbon isotopes, except for sample C40, which used ${ }^{13} \mathrm{C}$ enriched graphite.

More specifically, the carbon-doped hBN crystals were precipitated from the molten metal solution in a high temperature single-zone furnace. For growing the crystals listed in the first column of Table 1, the precursor powders with compositions listed in the $2^{\text {nd }}-4^{\text {th }}$ columns 


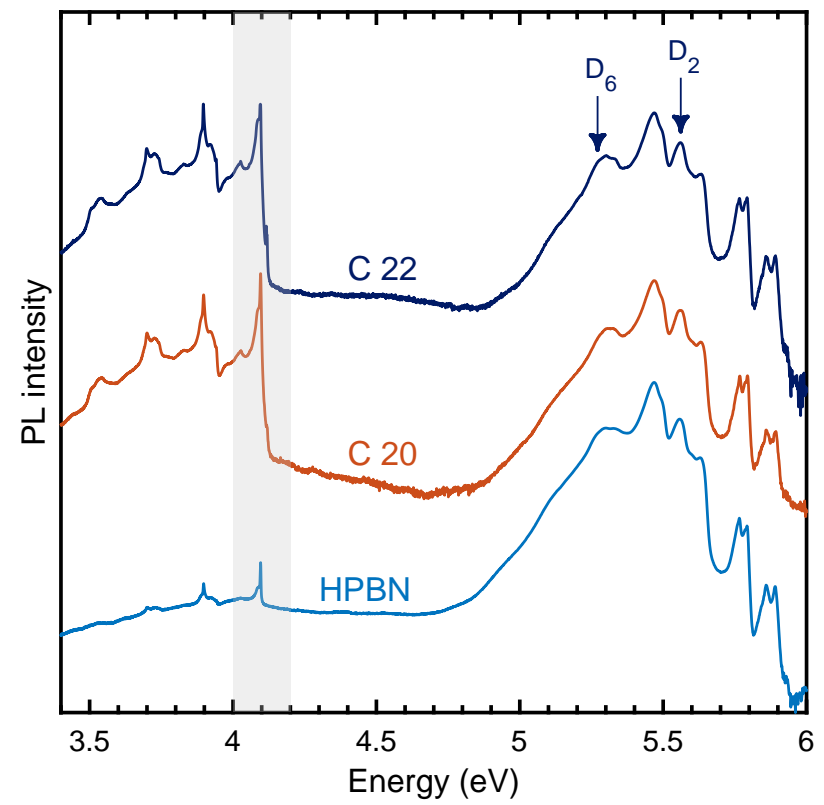

FIG. 1. Macro-PL spectrum at $8 \mathrm{~K}$ (semi-log scale), with an excitation energy of $6.35 \mathrm{eV}$, in bulk hBN synthesized either with iron (C22) or nickel (C20), and the hBN powder used as precursor, here in the form of Hot Pressed Boron Nitride (HPBN).

were loaded in either an alumina or a HPBN ceramic crucible, and transferred into the furnace. The furnace was evacuated and purged with the atmospheric gases listed in the $6^{\text {th }}$ column multiple times to remove all residual oxygen. For growing pure hBN crystals, a small amount of hydrogen is typically added with the flowing nitrogen, to reduce and remove any oxides from the source materials. However, to avoid excessive etching of the graphite source for growing these carbon-doped hBN crystrals, carbon monoxide was used instead of hydrogen, as it reacts with, and removes oxygen, but not carbon. The precursor powders were heated up to $1550^{\circ} \mathrm{C}$ to form liquid phase and held at this temperature for 24 hours to thoroughly dissolve the carbon, boron and nitrogen into the molten metal solution. The crystals were then grown by cooling the system to $1300^{\circ} \mathrm{C}$ at rates of $4^{\circ} \mathrm{C} /$ hour or $0.5^{\circ} \mathrm{C} /$ hour, as listed in the $5^{\text {th }}$ column. During crystal growth, the atmospheric gases were continuously flowed to the system at the rate of $125 \mathrm{sccm}$ and the system was kept at 850 torr. After crystal growth, the system was then quenched to room temperature at $50^{\circ} \mathrm{C} /$ hour.

\section{Iron vs nickel as solvent}

As alluded to earlier, the solubility of boron is higher in nickel than iron while the residual amount of carbon impurities in our Fe is smaller than in our Ni. PL is an efficient method to diagnose semiconductors [22] there- fore we use it to get information regarding the structural properties and residual dopings of these BN crystals. For that reason we begin by comparing the $8 \mathrm{~K}$ PL features in a wide spectral range between 3.5 and $6 \mathrm{eV}$ [Fig.1], recorded in cases of samples $\mathrm{C} 20$ (Fe-Cr solvent) and $\mathrm{C} 22$ (Ni-Cr solvent), and of the BN powder used as precursor, here in the form of HPBN. The PL spectrum of the $\mathrm{BN}$ precursor displays emission lines near $4 \mathrm{eV}$ (shaded region in Fig.1). These lines are also found in the PL spectra of C20 and C22. These lines form a more or less documented series, depending on the growth run, with in general, the detection of a strong feature at $4.1 \mathrm{eV}$. We emphasise that these sharp lines were never recorded in case of samples grown using the protocol of Ref.[23], which led us to revealing the optical performances of mono-isotopically boron-purified BN samples [24]. For these growths, $\mathrm{B}$ and $\mathrm{N}_{2}$ where used instead of a $\mathrm{BN}$ powder.

PL intensities have been plotted logarithmically in Fig.1. The PL spectrum below $5 \mathrm{eV}$ corresponds to the recombination lines arising from deep levels. There is the well-known zero-phonon line (ZPL) at $4.1 \mathrm{eV}$ with its accompanying phonon replicas at lower energies [13, 25]. Because of the heavy carbon-doping in our hBN crystals, the PL intensity of the $4.1 \mathrm{eV}$ line is comparable to that of the stacking faults around $5.5 \mathrm{eV}$ [Fig.1], in contrast to the peak observed in powder samples, if it is seen at all. This is in agreement with similar studies reported in Ref.[13] that led those authors to relate the $4.1 \mathrm{eV}$ line to carbon/oxygen impurities. In Fig.1, the PL line shape is not influenced by the chemical composition of the solvent (iron or nickel) within the inhomogeneous linewidth of our hBN crystals. It is important to emphasize the occurrence of a doublet feature centered at $4.12 \mathrm{eV}$, with an inter-level splitting of $\sim 2 \mathrm{meV}$ in sample $\mathrm{C} 22$ (grown using $\mathrm{Cr}-\mathrm{Ni}$ ) which is also recorded but weaker in sample C20 (grown using Cr-Fe), as shown in Fig.2. The PL feature of the BN powder displayed in Fig.2 indicates that it is also present in the precursor. Therefore this doublet line is correlated to a contaminant contained in the BN precursor. It has nothing to do with the contamination of $\mathrm{Fe}, \mathrm{Ni}$ or $\mathrm{Cr}$.

Finally, we observe a broad composite fluorescence band in the visible portion of the spectra (not shown here). There have been many experimental studies of defect-related single photon sources operating in the visible spectrum with recent interpretations suggesting lattice related defects $[10,26-28]$. The only difference between $\mathrm{Ni}$ and $\mathrm{Fe}$ as a solvent is the unsystematic recording of a weak and broad PL band at $4.6 \mathrm{eV}$ in case of using the Ni-Cr solvent. We thus conclude that the use of $\mathrm{Ni}$ or $\mathrm{Fe}$ has only a marginal influence on the optoelectronic properties of our hBN crystals.

In the following, we focus on the impact of carbondoping considering separately shallow levels in the 5-5.7 $\mathrm{eV}$ range (Section III), and deep levels around $4.1 \mathrm{eV}$ 


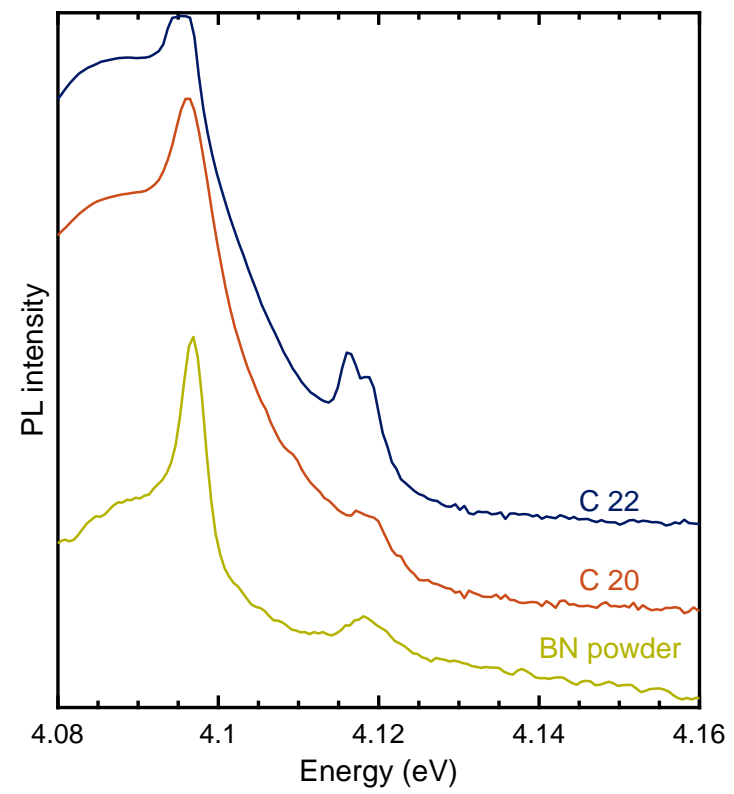

FIG. 2. Zoom in the $4 \mathrm{eV}$ region (linear scale) of the macro-PL spectrum in bulk hBN synthesised either with iron (C20) or nickel (C22), together with a hBN powder used as precursor (BN Powder).

\section{(Section IV)}

\section{SHALLOW LEVELS}

Fig.3 displays (on a semi-logarithmic scale) the PL spectra recorded at $8 \mathrm{~K}$ for different samples: (from top to bottom) a test un-doped hBN crystal purchased from HQ Graphene (HQG), samples C22 and C27 (w/o CO), and sample $\mathrm{C} 33$ (w/CO but with different $\mathrm{C} / \mathrm{BN}$ ratios, see Table 1).

The 5.7-6 eV energy range corresponds to the intrinsic part of the emission spectrum. This series of lines correspond to the well-defined phonon-assisted optical transitions typical of indirect band-gap semiconductors and here, of the hexagonal BN stacking, with their specific spectral distribution [29]. Zooming in on each of these phonon-assisted recombination lines reveals a similar lowenergy fine structure, due to the emission of phonon overtones involving the low-frequency Raman active mode at zone center [30].

Below the energy of the LO-assisted recombination $(5.75 \mathrm{eV})$ starts the spectral range of defect-related emission. From $5.7 \mathrm{eV}$ down to $5 \mathrm{eV}$, there is a series of discrete lines split from each other by about $147 \mathrm{meV}$. Transverse optical phonons at the $\mathrm{K}$ point of the Brillouin zone assist inter- $\mathrm{K}$ valley scattering, producing a series of discrete recombination bands because the stacking faults in bulk hBN $[31,32]$ provide a density of final electronic states in the Fermi's golden rule, and shape the envelope of the PL spectrum [33]. Two distinct lines are also detected that we labelled D2 and D6 [Fig.1] in our

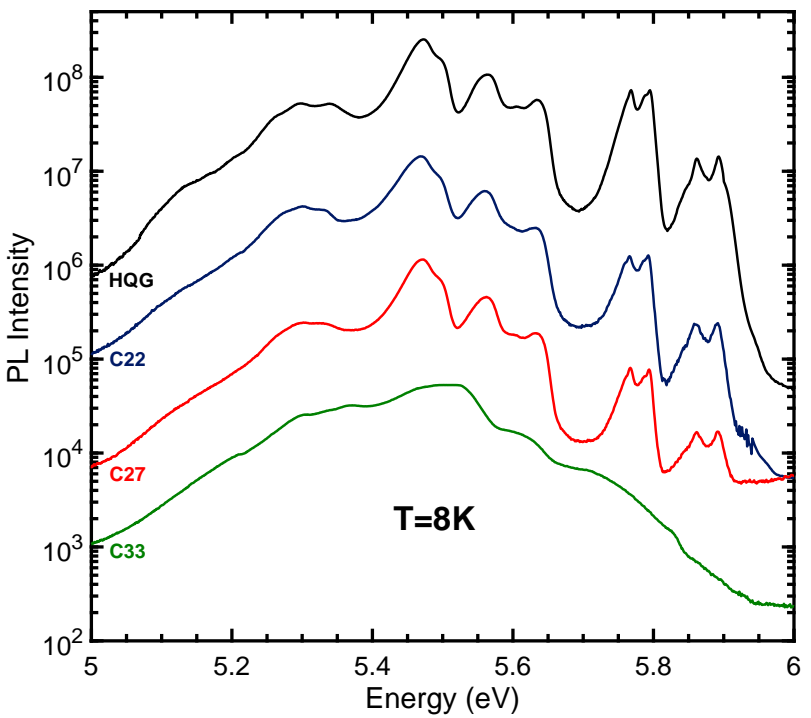

FIG. 3. PL spectra recorded at $8 \mathrm{~K}$ in bulk hBN crystals for energies ranging between 5 and $6 \mathrm{eV}$ (semi-log scale). Spectra are vertically shifted for clarity. Growth conditions are given in Table 1. HQG stands for a test un-doped hBN crystal purchased from HQ Graphene.

initial paper [33], where we tentatively attributed them to the BN di-vacancy following Ref.[18].

The PL spectrum is almost identical for the first three samples (HQG, C22, and C27). In contrast, in the heavily-doped C33 sample, the PL spectrum presents less defined features. The sharp and detailed series of phonon-assisted recombination lines above $5.7 \mathrm{eV}$ is strongly broadened and hardly observable and the defectrelated emission lines are also broadened. More importantly we note an apparent blue-shift of the most intense line to $5.52 \mathrm{eV}$ in $\mathrm{C} 33$, shifting from $5.47 \mathrm{eV}$ in the other samples.

The higher carbon concentration in C33 may affect the crystal quality and result in a decrease of the intensity of the intrinsic recombination lines together with a larger inhomogeneous broadening, which would contribute to washing out the series of phonon replicas above $5.7 \mathrm{eV}$. As a matter of fact, this may also impact the defectrelated emission lines, but we note a distortion of the peak around $5.5 \mathrm{eV}$. In HQG, C22, and $\mathrm{C} 27$, the energy of the maximum is $5.47 \mathrm{eV}$ with a high-energy shoulder at $5.5 \mathrm{eV}$ [Fig. 3] whereas we observe a broader asymmetric line in $\mathrm{C} 33$ with a maximum at $5.52 \mathrm{eV}$, an energy that corresponds to a minimum of the PL spectrum in HQG, $\mathrm{C} 22$, and $\mathrm{C} 27$. Finally, a secondary maximum appears at $5.37 \mathrm{eV}$ in $\mathrm{C} 33$, as a high-energy shoulder of the $5.3 \mathrm{eV}$ band, again at an energy where the PL spectrum of HQG, $\mathrm{C} 22$, and $\mathrm{C} 27$ displays a minimum. These observations suggest the existence of new transitions in the optical response of heavily carbon-doped hBN crystals.

To test this hypothesis, we compare in Fig. 4 the PL 


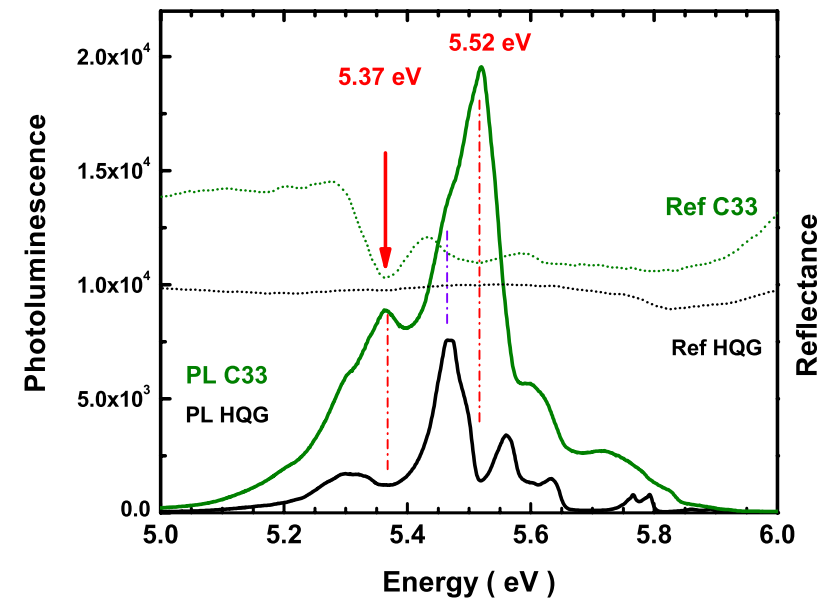

FIG. 4. PL (solid lines) and reflectance (dotted lines) spectra on linear scales, recorded in a test un-doped hBN crystal (HQG, black lines) and a heavily carbon-doped one (C33, green lines) at $10 \mathrm{~K}$. Note the coincidences between the reflectance minima and the PL maxima in sample C33.

and reflectance spectra (on linear scales) recorded in HQG and C33. In the former case, the reflectivity (dotted black line in Fig.4) displays smooth variations with a rather flat spectrum in the spectral domain where the PL spectrum (solid black line in Fig.4) is on the contrary very rich with many lines due to intervalley scattering into excitonic states bound to stacking faults, as explained above. Such a difference between the spectra is fully consistent with the distinct nature of the microscopic processes involved in PL and reflectivity. While reflectivity probes a material in its thermodynamic equilibrium, PL results from a non-equilibrium state where carrier relaxation and thermalization play a key role. The presence of many defect-related lines around $5.5 \mathrm{eV}$, in the PL spectrum but not in the reflectance one, is a genuine signature of relaxation effects with resonances of intervalley scattering, further supporting the interpretation initially proposed in Ref.[33].

The heavy carbon-doping in C33 not only modifies the PL spectrum (solid green line in Fig.4) but also the reflectance one (dotted green line in Fig.4). Strikingly, there are pronounced reflectance oscillations, in resonance with the new emission lines at 5.37 and $5.52 \mathrm{eV}$, pointed out above. This observation confirms the existence of new optical transitions associated with heavy carbon-doping in hBN crystals. The incorporation of carbon during the growth process produces complementary states with a high enough density and oscillator strength of their optical transition to significantly change the dielectric constant in the $5.3 \mathrm{eV}$ to $5.6 \mathrm{eV}$ energy region.

Following Ref.[18], we tentatively attribute the lines at 5.37 and $5.52 \mathrm{eV}$ to an exciton bound to the defect formed by a carbon substituting a nitrogen $\left(\mathrm{C}_{\mathrm{N}}\right)$. In Ref.[18], the optical absorption spectrum of hBN calculated by the Bethe-Salpeter equation predicted that native point defects or impurities induce novel optical transitions close to the band-gap, corresponding to defect bound excitons. The heavy carbon-doping in $\mathrm{C} 33$ makes the $\mathrm{C}_{\mathrm{N}}$-bound exciton a strong candidate for the 5.37 and $5.52 \mathrm{eV}$ lines.

\section{DEEP LEVELS}

In this section, we focus on emission lines at lower energy than in the previous part of our paper. In $\mathrm{hBN}$, the emission lines below $5 \mathrm{eV}$ come from deep levels due to intrinsic or extrinsic point defects in the hBN lattice. In Section IV A, we first discuss the well known $4.1 \mathrm{eV}$ line and our original measurements in hBN crystals doped with ${ }^{13} \mathrm{C}$, and then address in Section IV B the additional lines in the 4.1-4.2 eV range, which are not discussed in the literature.

\section{A. The $4.1 \mathrm{eV}$ line}

Carbon was early suggested as the possible origin of the $4.1 \mathrm{eV}$ emission in $\mathrm{hBN}[11,12]$. It was later reported that the intensity of the $4.1 \mathrm{eV}$ line is strongly enhanced in hBN crystals with a high carbon concentration [13]. Advanced spectroscopic studies have been devoted to the vibronic spectrum and the particular structure of the phonon sidebands [25], or to isolated single centers in cathodoluminescence experiments [34]. However, there is so far no definitive proof that carbon enters into the composition of the point defect leading to the $4.1 \mathrm{eV}$ line. Moreover, recent papers reported no positive correlation between the carbon concentration and the intensity of the $4.1 \mathrm{eV}$ emission [14], and excluded $\mathrm{C}_{\mathrm{N}}$ as its origin on the basis of density functional theory with a hybrid functional [19]. Other possible origins have been proposed in Ref.[15], such as a $\mathrm{V}_{\mathrm{B}} \mathrm{O}_{\mathrm{N}}$ complex.

In this controversial context, we have grown hBN crystals with isotopically-controlled carbon doping in order to further test if carbon is a constituent of the defect emitting at $4.1 \mathrm{eV}$. More specifically, we have performed high-resolution measurements of the energy of the ZPL in $\mathrm{hBN}$ crystals doped either with ${ }^{13} \mathrm{C}$ (sample C40, Table 1) or with ${ }^{\text {nat }} \mathrm{C}$ (sample $\mathrm{C} 27$, Table 1), i.e. the natural mixture of $\sim 99 \%$ in ${ }^{12} \mathrm{C}$ and $\sim 1 \%$ in ${ }^{13} \mathrm{C}$ ( ${ }^{\text {nat }} \mathrm{C}$ being close to pure $\left.{ }^{12} \mathrm{C}\right)$.

Isotopic purification is a powerful tool in semiconductor physics enabling the fabrication of chemically and isotopically pure crystals and crystals doped with isotopically pure impurities [35]. For point defects, isotopic control of the atoms forming the host lattice impacts the vibronic part of the emission spectrum with an isotopic shift of the phonon sideband, while isotopic selectivity of the defect itself can shift the zero-phonon lines. Several mechanisms may explain this isotopic shift, such as 

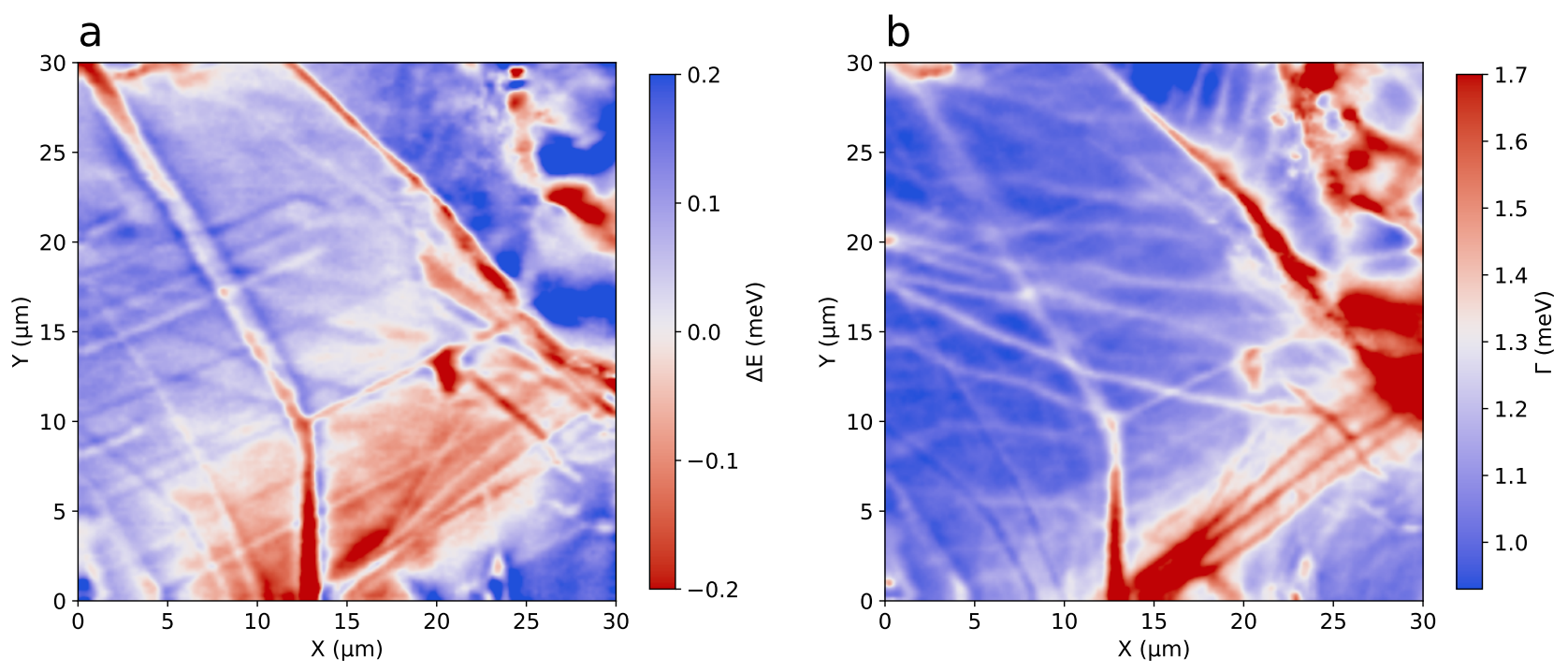

FIG. 5. Spatial variations of the shift $\Delta \mathrm{E}$ (a) and broadening $\Gamma$ (b) of the $\mathrm{ZPL}$ in a $30 \times 30 \mu \mathrm{m}^{2}$ region of sample $\mathrm{C} 40$ at $10 \mathrm{~K}$. The average ZPL energy is $4.0968 \mathrm{eV}$.

a modification of the confinement or the electron-phonon interaction [35]. Quantitatively, the energy shifts are very small. For the boron-bound exciton in silicon, the isotopic shift is of the order of $1 \mu \mathrm{eV}$ when switching from ${ }^{10} \mathrm{~B}$ to ${ }^{11} \mathrm{~B}[35]$, but it can be much larger as for G-centers in silicon with an isotopic shift of $\sim 15 \mu \mathrm{eV}$ between ${ }^{12} \mathrm{C}$ and ${ }^{13} \mathrm{C}$ doping [36], or even $\sim 600 \mu \mathrm{eV}$ between ${ }^{28} \mathrm{Si}$ and ${ }^{30} \mathrm{Si}$ for the SiV center in diamond [37].

In the case of $\mathrm{hBN}$ doped either with ${ }^{\text {nat }} \mathrm{C}$ or ${ }^{13} \mathrm{C}$, the search for an isotopic shift of the $4.1 \mathrm{eV}$ line requires highspectral resolution measurements. Moreover, one has to pay attention to possible variations of the ZPL energy within a given sample before comparing ${ }^{\text {nat }} \mathrm{C}$ - and ${ }^{13} \mathrm{C}$ doped hBN in order to exclude any artefact due to a shift not coming from the isotopically-controlled doping.

In the next section (IV A 1), we first study the spatial variations of the energy and linewidth of the ZPL in sample C40, and then compare in section IV A 2 the statistical analysis of our data recorded in ${ }^{\text {nat }} \mathrm{C}$ - and ${ }^{13} \mathrm{C}$ doped hBN crystals (samples C27 and C40, Table 1).

\section{Spatial inhomogeneity}

Fig.5 displays the analysis of a set of data measured by means of our UV scanning confocal micro-PL setup (Section II A 3) in a $30 \times 30 \mu \mathrm{m}^{2}$ region of sample C40 at $10 \mathrm{~K}$. For each of the $150 \times 150$ positions, we acquired the $\mathrm{PL}$ spectrum over an integration time of $3 \mathrm{~s}$, resulting in a total acquisition of 27 hours for the full scan. The ZPL was then fitted with a Gaussian function for each point. With $\sim 5.10^{4}$ counts at the maximum of the ZPL, the standard deviation for the estimation of the ZPL energy and width was $6 \mu \mathrm{eV}$ and $10 \mu \mathrm{eV}$, respectively. Compared to the typical shifts discussed above, we conclude that our acquisition parameters are a priori adapted for resolving isotopic effects in $\mathrm{hBN}$.

In Fig.5(a) we show the energy shift $\Delta \mathrm{E}$ of the ZPL relative to the average value of $4.0968 \mathrm{eV}$ in this region. The variations of the ZPL Full Width at Half Maximum (FWHM) $\Gamma$ are plotted in Fig.5(b). The first finding is that the fluctuations of the ZPL energy over the $30 \times 30 \mu \mathrm{m}^{2}$ scanned region have an amplitude as large as one meV. These fluctuations contribute to the inhomogeneous broadening of the $4.1 \mathrm{eV}$ line. With an average FWHM on the order of $1.3 \mathrm{meV}$ [Fig.5(b)], the \pm 0.2 $\mathrm{meV}$ variations of the ZPL energy are consistent with the $1.9 \mathrm{meV}$ value of the ZPL width previously reported by macro-PL measurements in Ref.[25], but here we notably demonstrate the sub- $\mu \mathrm{m}$ scale at which the ZPL energy varies.

Interestingly, the ZPL shifts [Fig.5(a)] appear spatially-correlated with the ZPL broadening [Fig.5(b)]. Although there is not a one-to-one correlation, the overall pattern is the same in Fig.5(a)\&(b), with lines of different orientations in close analogy to the luminescence maps recorded by cathodoluminescence [31]. We interpret these lines as folds or extended defects in the lamellar structure of our hBN crystal.

The spatial correlation of the ZPL shift and broadening suggests they correspond to the real and imaginary parts of the same perturbation. That they also have the same magnitude (in the $0.1 \mathrm{meV}$ range here) further supports a common origin, presumably mechanical strain related to the stacking faults in $\mathrm{hBN}$.

Maps of the Raman-active mode at $\sim 1370 \mathrm{~cm}^{-1}$ (170 $\mathrm{meV}$ ) over the same area were featureless maps (not shown here) with random fluctuations in the range of \pm $60 \mu \mathrm{eV}$. This may come from the reduction of the stressinduced energy shift for phonons compared to electrons 
$[38,39]$.

Although detrimental to our objective of resolving an isotopic shift in ${ }^{\text {nat }} \mathrm{C}$ - and ${ }^{13} \mathrm{C}$-doped $\mathrm{hBN}$ crystals, the sensitivity of the ZPL energy to the presumable perturbation caused by strain is an important piece of information for elucidating the structure of the point defect leading to the $4.1 \mathrm{eV}$ emission. In this context, PL experiments as a function of pressure could provide interesting complementary inputs [40] for identifying the nature of the defect and reaching a quantitative interpretation of our measurements.

$$
\text { 2. }{ }^{13} C \text { vs }{ }^{\text {nat }} C \text { doping }
$$

Keeping in mind the spatially-resolved ZPL fluctuations in Fig.5, we now compare the ZPL energy in hBN crystals doped either with ${ }^{\text {nat }} \mathrm{C}$ and ${ }^{13} \mathrm{C}$. Fig.6 shows the probability density of detecting the ZPL at a given energy. Following the data analysis described in the previous section, we have computed the statistical distributions of the ZPL energy in two $30 \times 30 \mu \mathrm{m}^{2}$ regions, arbitrarily selected in each crystal.

We first note the different shapes and widths of the four statistical distributions, which can be narrow or asymmetric in the two types of samples. More importantly, there is no spectral separation indicative of an isotopic shift of the ZPL, within the inhomogeneous broadening of the $4.1 \mathrm{eV}$ line. Moreover, there does not seem to be any specific feature of the spatial fluctuations of the ZPL energy from ${ }^{13} \mathrm{C}$ - to ${ }^{\text {nat }} \mathrm{C}$-doped $\mathrm{hBN}$ crystals. As explained in the previous section, this suggests mechanical strain impacts the ZPL position independently of the carbon isotopes.

We thus conclude that there is either no isotopic shift, or a finite one but smaller than the inhomogeneous broadening. Isotopic selectivity in carbon-doping has not brought any proof to confirm or infirm the presence of carbon in the structure of the defect emitting at $4.1 \mathrm{eV}$. However, our test demonstrated that the spatial fluctuations of the ZPL were in a sub- $\mu \mathrm{m}$ scale. The correlation of the ZPL shift and broadening indicates a common origin, with original quantitative measurements which hopefully will be useful for testing various models in future calculations.

In the last part of our paper, we finally present our PL experiments dealing with additional spectral lines in the 4.12-4.16 range, which, in contrast to the $4.1 \mathrm{eV}$ line, are not discussed in the literature.

\section{B. The 4.12-4.16 eV lines}

The $4.1 \mathrm{eV}$ line is not the only feature related to deep levels in the UV spectral range. The existence of additional lines at energies higher than $4.1 \mathrm{eV}$ was never

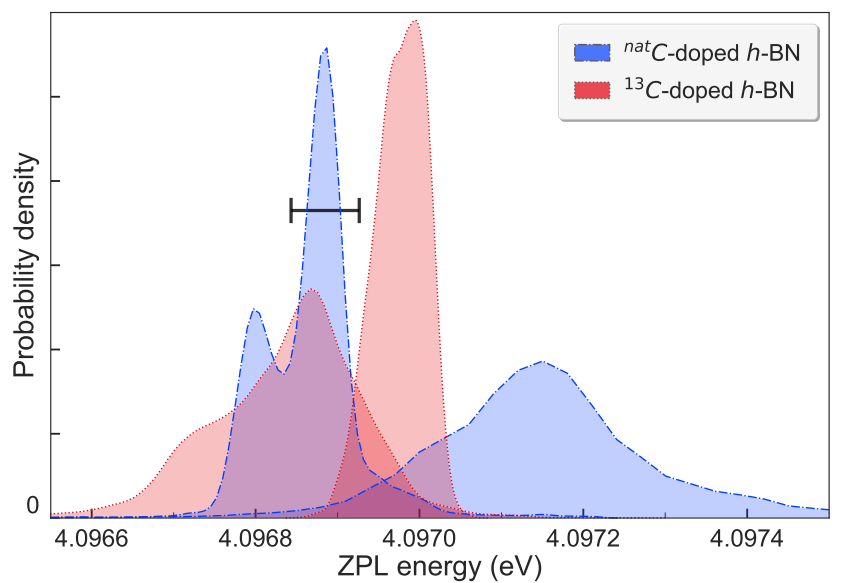

FIG. 6. Statistical analysis of the ZPL energy in ${ }^{13} \mathrm{C}$ - and ${ }^{\text {nat }} \mathrm{C}$-doped $\mathrm{hBN}$ crystals: the normalized probability density of the ZPL energy is plotted for four $30 \times 30 \mu \mathrm{m}^{2}$ regions; two in a ${ }^{13} \mathrm{C}$-doped crystal (C40, red data), two in a ${ }^{\text {nat }} \mathrm{C}$-doped crystal (C27, blue data). The $100 \mu \mathrm{eV}$-large horizontal bar indicates the difference of the mean values of the ZPL energy in ${ }^{13} \mathrm{C}$ - and ${ }^{\text {nat }} \mathrm{C}$-doped $\mathrm{hBN}$ crystals, the mean values being calculated for a total of ten $30 \times 30 \mu \mathrm{m}^{2}$ regions.

pointed out nor discussed in the literature, to the best our knowledge. In the following, we present extensive characterization of the additional lines in the 4.12-4.16 range, by means of macro-PL and reflectance experiments (Section IV B 1) and spatially-resolved micro-PL measurements (Section IV B 2). We show that their PL intensity increases in heavily carbon-doped $\mathrm{hBN}$ crystals and that all the additional lines are independent of each other.

\section{Macro-PL and reflectance experiments}

Fig.7 displays the PL spectra of three samples in the 3.5 to $4.25 \mathrm{eV}$ spectral range. Emission from the $4.1 \mathrm{eV}$ deep level is always recorded with its sharp ZPL, phonon sidebands and prominent optical phonon replicas at 3.9 and $3.7 \mathrm{eV}[25,41]$. A new line at $4.12 \mathrm{eV}$ (later called UV1) is observed with a doublet fine structure splitting, as seen earlier in Fig. 2 and better resolved below in micro-PL measurements. In sample $\mathrm{C} 27$, there are additional weak lines at 4.14 and $4.16 \mathrm{eV}$ (called UV2 and UV3, respectively), all three of these lines being much more intense in the heavily carbon-doped C33 sample. We also note that all of these lines broaden when increasing the amount of carbon involved into the growth protocol, which likely prevents us from observing any fine structure splitting, if any, for UV2 and UV3. Finally, phonon replicas of UV1, UV2, and UV3 are detected, readily observed and red-shifted by $200 \mathrm{meV}(\mathrm{LO}(\Gamma)$ phonon) and $400 \mathrm{meV}(2 \mathrm{LO}(\Gamma)$ phonons) from their ZPL [Fig.7]. 


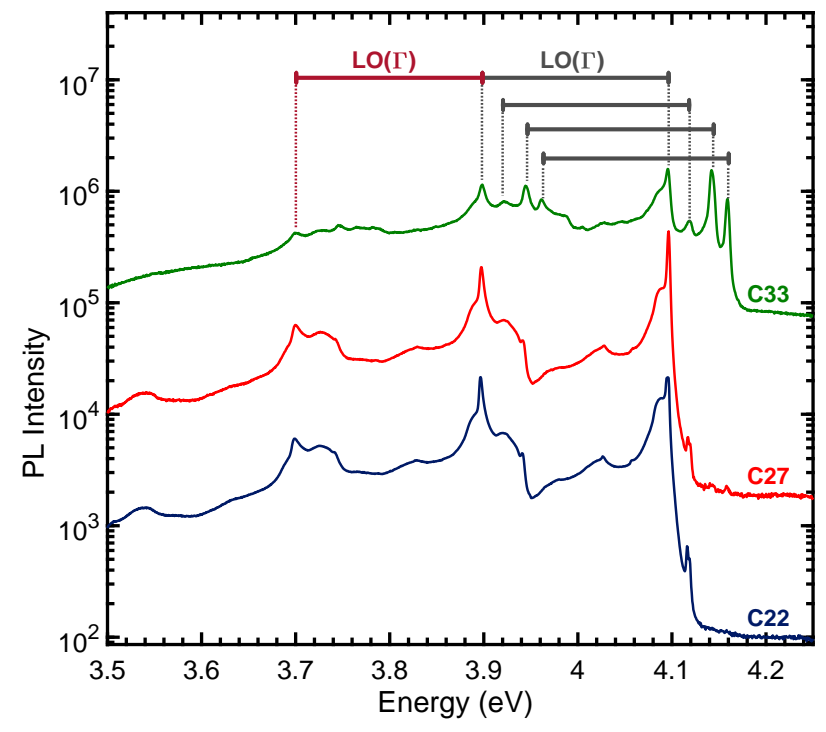

FIG. 7. Full set of the PL spectra (semi-log scale) of carbondoped hBN samples in the 3.5 to $4.25 \mathrm{eV}$ range. The spectra are vertically shifted for clarity. Note the occurrence of new lines at higher energy than $4.1 \mathrm{eV}$. The horizontal bars have a $200 \mathrm{meV}$ width, corresponding to the $\mathrm{LO}(\Gamma)$ phonon energy.

The first indication that UV1-3 do not share the same microscopic origin as the $4.1 \mathrm{eV}$ deep level comes from the vibronic part of their PL spectrum. The experimental data recorded in C33 are plotted on a linear scale in Fig. 8 in order to better resolve the different lineshapes of UV1-3 compared to the $4.1 \mathrm{eV}$ line. At low temperature [8K, top spectrum in Fig.8], we can distinguish the ZPL and the low-energy sideband in the emission spectrum of the $4.1 \mathrm{eV}$ defect as described in Ref.[25], although the carbon-doping leads to broadening of the ZPL. On the contrary, for UV1-3, no phonon sideband is observed and each of the UV1-3 lines can be fairly fitted to a symmetric profile, with a Gaussian function. Moreover, on raising the temperature [Fig.8], the FWHM of UV1-3 increases from $6 \mathrm{meV}$ at $8 \mathrm{~K}$ to $9 \mathrm{meV}$ at $120 \mathrm{~K}$. Such a temperature dependence contrasts with the phenomenology discussed in Ref.[25] where we observed a $5.5 \mathrm{meV}$ broadening in the $8-120 \mathrm{~K}$ range, further suggesting a different microscopic structure in UV1-3 compared to the $4.1 \mathrm{eV}$ defect.

Furthermore, looking at the PL signal intensity as a function of temperature [Fig.8] we do not record any cross-talk between the lines, with a constant intensity ratio among the set of optical transitions in the 3.8 to $4.2 \mathrm{eV}$ range. This observation indicates the absence of interlevel crossing in the relaxation dynamics, either because the spatial separation of the defects is larger than the carrier diffusion length, or because they are formed in distinct mesoscopic domains due to the local fluctuations of the growth conditions during the synthesis of the hBN crystals.

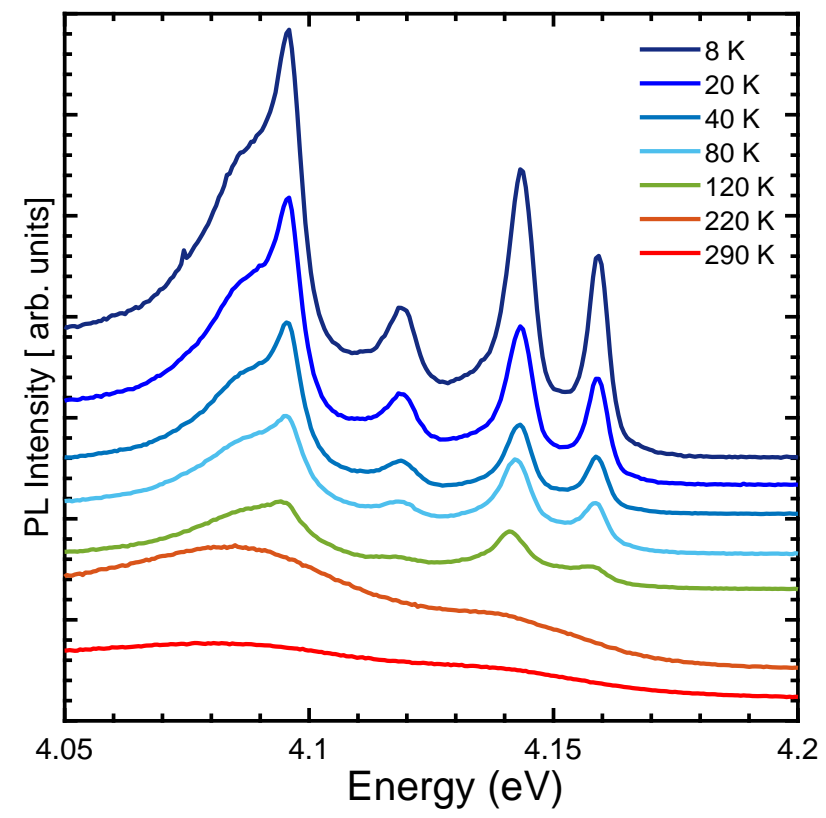

FIG. 8. Temperature-dependent PL measurements (linear scale) in the heavily carbon-doped $\mathrm{hBN}$ sample $\mathrm{C} 33$, in the 4.05 to $4.2 \mathrm{eV}$ range. The spectra are vertically shifted for clarity.

Direct access to the oscillator strength of the optical transitions is obtained by reflectivity experiments around $4.1 \mathrm{eV}$. In Fig.9, we compare the PL and reflectance spectra recorded at $8 \mathrm{~K}$ in sample $\mathrm{C} 33$. The reflectivity signal exhibits a marked peak at $4.1 \mathrm{eV}$, testifying to a substantial oscillator strength. Because of the mirror symmetry between phonon-assisted absorption and phonon-assisted emission, we record a series of blue-shifted minima that are the high-energy analogues of the phonon replicas detected in the PL spectrum [Fig.9], with an inter-level spacing of $200 \mathrm{meV}$ as discussed above. Note the ZPL is broader in reflectance than in PL, thus smearing out the fine structure of the phonon replicas in reflectivity.

Surprisingly, nothing is detected for the UV1-3 deep levels in reflectivity. Although the PL lines of UV1-3 and the $4.1 \mathrm{eV}$ defect are of comparable intensities in this sample, no reflectance minima are seen at the energies of the new carbon-related PL lines. This effect may come from either a smaller oscillator strength, or a lower density of the UV1-3 point defects. In both cases, the interpretation of the high PL signal intensity from UV1-3 implies a more efficient carrier relaxation in these novel carbon-related point defects.

To get further insight in these deep levels, we present in the final part of our paper measurements by means of our UV scanning confocal micro-PL setup. 


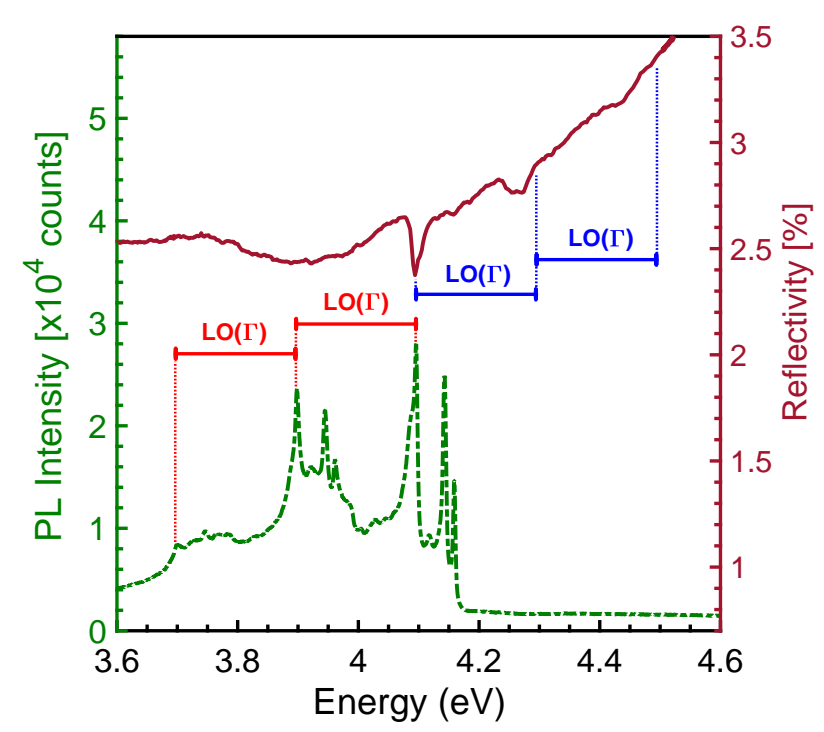

FIG. 9. PL (green dashed line) and reflectivity (purple solid line) spectrum on linear scales, recorded at $8 \mathrm{~K}$ for sample C33 in the $4 \mathrm{eV}$ region. The horizontal bars have a $200 \mathrm{meV}$ width, corresponding to the $\mathrm{LO}(\Gamma)$ phonon energy. They are displayed in red for the red-shifted phonon-assisted emission (below $4.1 \mathrm{eV}$ ), and in blue for the blue-shifted phonon-assisted absorption (above $4.1 \mathrm{eV}$ ).

\section{Micro-PL experiments}

In order to get further insight in these deep levels, we finally present measurements by our UV scanning confocal micro-PL setup. We recall these measurements consist in recording the PL spectrum at each point of a given region that is scanned at the surface of the sample. We first studied the spatial variations of the UV1-3 PL signal intensity recorded by means of our UV scanning confocal microscope in sample C27, at $10 \mathrm{~K}$. Carbon-doping in this hBN crystal is moderate to minimise broadening of the PL lines. In Fig.10(a), the PL map is plotted after integration of the signal intensity from 4.112 to 4.175 $\mathrm{eV}$ in order to better visualize the presence of the novel carbon-related UV1-3 lines. With this color code related to a spectral range excluding the $4.1 \mathrm{eV}$ defect, we stress the regions appearing dark in Fig.10(a) correspond to a large PL intensity of $\sim 5.10^{3}$ counts per second for the 4.1 eV ZPL. In terms of the intensity of the UV1-3 lines, Sample C27 is thus more or less homogeneously dim except for very bright domains. The $4.1 \mathrm{eV}$ defect is also rather uniform and intense with a local increase in some bright regions of Fig.10(a), as detailed below.

The PL spectra of five representative regions are provided in the lower panels (b-f) of Fig.10. In the homogeneous part of the sample [circle 1 in Fig.10(a)], only the $4.1 \mathrm{eV}$ line is detected with a sharp ZPL and the lowenergy acoustic phonon sideband [Fig.10(b)]. We note the high signal-to-noise ratio with a dynamical range of three decades between the maximum of the ZPL at 4.1 $\mathrm{eV}$ and the noise level at higher energy. Such a precision

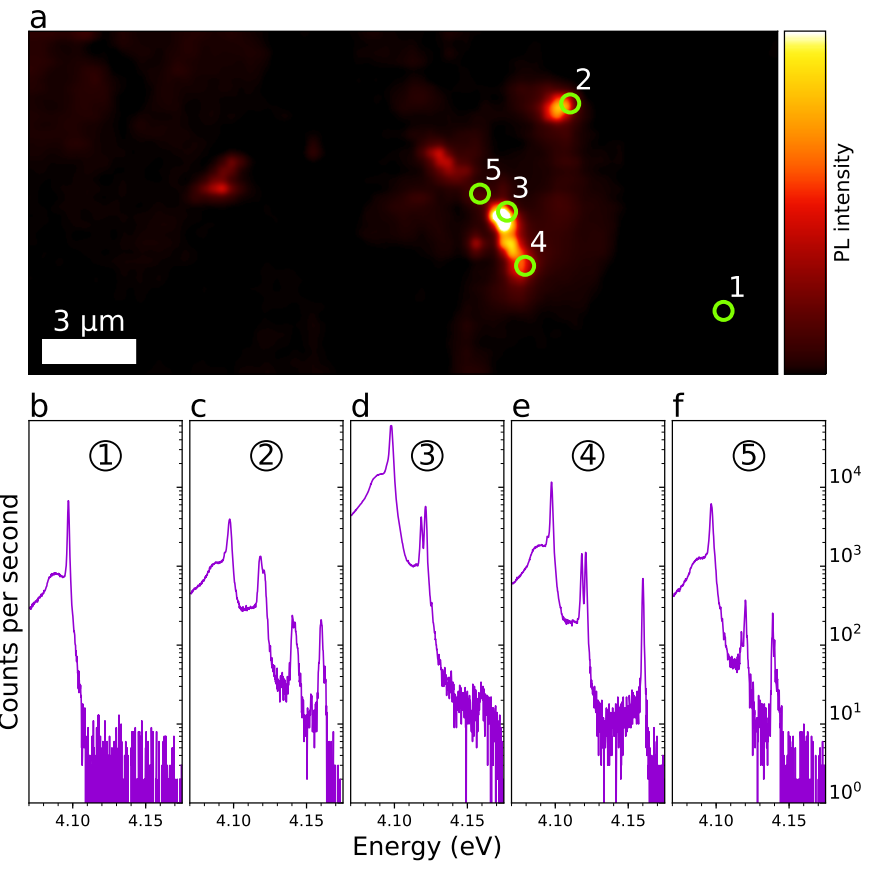

FIG. 10. (a) Spatial variations of the PL signal intensity recorded by a UV scanning confocal microscope in sample $\mathrm{C} 27$, at $10 \mathrm{~K}$, for an excitation power of $0.35 \mathrm{~mW}$. The PL signal is integrated from 4.112 to $4.175 \mathrm{eV} .5 \mathrm{~s}$ integration time per acquisition point. (b-f) PL spectrum on a log scale recorded at the five different locations indicated by circles in (a).

is important in view of claiming the contribution of the novel carbon-related UV1-3 lines is negligible within our signal-to-noise ratio.

Circles 2, 3 and 4 are located in the bright white regions. As expected, we observe that the UV1-3 lines have a PL signal intensity always lower than the $4.1 \mathrm{eV}$ defect, in agreement with the macro-PL measurements presented in Fig.7. Our spatially-resolved PL measurements reveal the striking absence of spatial correlation between the UV1-3 lines: all three are detected at point 2, only UV1 at point $3, \mathrm{UV} 1$ and UV3 at point 4 , and UV1 and UV2 at point 5. We thus conclude that the novel emission lines related to carbon-doping in $\mathrm{hBN}$ do not come from the same defect but that they originate from three independent defects. This is a crucial information and a key indication that one has to look for three novel microscopic configurations in order to account for the micro-PL measurements in Fig.10. It is very tempting, having in mind proposals that were made for porous silicon [42] on $\mathrm{ZnO}$ [43], to invoke complexes of the $\mathrm{O}_{\mathrm{N}} \mathrm{H}$ kind, or the $\mathrm{C}_{\mathrm{B}} \mathrm{H}$ one, as candidates for these lattice defects. Unfortunately, theoretical works dedicated to these specific defects have not been performed yet.

Eventually, we point out the doublet structure of UV1, which is much better resolved in Fig.10 than in Fig.2. 


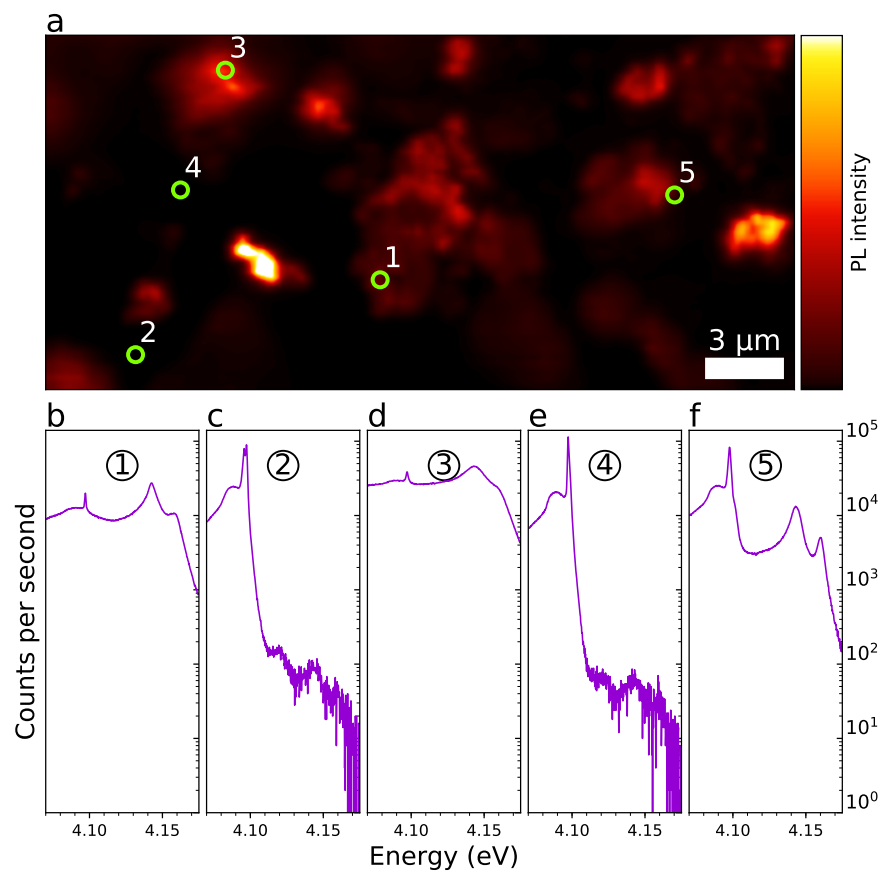

FIG. 11. (a) Spatial variations of the PL signal intensity recorded by a UV scanning confocal microscope in the heavily carbon-doped sample C33, at $10 \mathrm{~K}$, for an excitation power of $0.35 \mathrm{~mW}$. The PL signal is integrated from 4.112 to 4.175 eV. $2.5 \mathrm{~s}$ integration time per acquisition point. (b-f) PL spectrum on a log scale recorded at the five different locations indicated by circles in (a).

Thanks to the low carbon doping and correspondingly narrow defect lines, as well as the spatial resolution of our microscope, the spectra recorded in positions 3 and 4 show that UV1 is a doublet with a splitting of $2.7 \mathrm{meV}$ and a FWHM of $1.7 \mathrm{meV}$ for the two components. On the contrary, we could not detect any fine structure for UV2 and UV3, supporting the picture that each emission line originates from a distinct defect.

Fig.11 was recorded in identical conditions as Fig.10 but in a heavily carbon-doped hBN crystal (sample C33). We first notice the increased inhomogeneity with many domains giving a large PL signal intensity for the UV1-3 lines. Moreover, there is a significant broadening with a typical FWHM of the order of $\sim 10 \mathrm{meV}$ compared to 1.7 measured in Fig.10(d\&e). Nevertheless, the $4.1 \mathrm{eV}$ defect maintains a narrow ZPL as seen in Fig.11(c\&e), not only where the UV1-3 lines are hardly observable, but also in locations where they are intense [Fig.11(f)] or even dominate the PL spectrum [Fig.11(b,d)]. This phenomenology suggests that the UV1-3 defects are not only independent from each other but also uncorrelated to the $4.1 \mathrm{eV}$ deep level. This raises fundamental questions on the exact influence of carbon which is clearly involved here: is carbon itself incorporated into the defect structure, and in which crystallographic configurations with 4 point defects to be explained ? Does carbon play only a side-effect and promote the formation of deep levels without any embedded carbon atom?

At the very end of Section IV devoted to deep levels, we are thus left with numerous novel issues fed by our measurements in heavily carbon-doped hBN samples. The detailed characterization of the novel deep levels emitting above $4.1 \mathrm{eV}$ shows we are dealing with a new class of point defects in hBN crystals, where carbon plays a key role. Since new shallow levels were observed in heavily carbon-doped hBN (Section III) and interpreted as excitons bound to $\mathrm{C}_{\mathrm{N}}$, one may wonder if the UV1-3 lines are themselves the true candidates for point defects made of carbon, and if, on the contrary, the $4.1 \mathrm{eV}$ defect is carbon-free. In future studies the combination of advanced optical spectroscopy with nanoscale electron microscopy appears as a promising strategy for unveiling the nature of the defects.

\section{CONCLUSION}

We have investigated shallow and deep levels in carbon-doped hBN crystals. The incorporation of carbon during the growth process produces new optical transitions at 5.37 and $5.52 \mathrm{eV}$, which are observed in both reflectance and PL spectra. These shallow levels are attributed to an exciton bound to the defect formed by a carbon substituting a nitrogen $\left(\mathrm{C}_{\mathrm{N}}\right)$. For the deep levels around $4 \mathrm{eV}$, we have first investigated if isotopic selectivity in carbon-doping modifies the energy of the wellknown ZPL at $4.1 \mathrm{eV}$. We conclude that there is either no isotopic shift due to ${ }^{13} \mathrm{C}$, or a finite one but smaller than the inhomogeneous broadening. We have further resolved the spatial fluctuations of the ZPL at $4.1 \mathrm{eV}$ on a sub- $\mu \mathrm{m}$ scale. The correlation of the ZPL shift and broadening indicates a common origin, presumably strain variations in the crystal. Finally, we have shown the existence of additional optical transitions at 4.12, 4.14 and $4.16 \mathrm{eV}$ to which carbon doping induces a strong increase in their PL signal intensity. Reflectance, temperature-dependent PL, and spatially-resolved micro-PL demonstrate they correspond to three deep levels that are independent of each other, and uncorrelated to the $4.1 \mathrm{eV}$ defect. Our detailed characterization of a new class of shallow and deep levels in carbon-doped hBN crystals provides a wealth of novel informations for identifying the influence of carbon in the growth of $\mathrm{hBN}$, and its direct or indirect implication in the formation of the crystal defects.

\section{Acknowledgments}

We gratefully acknowledge C. L'Henoret for his technical support at the mechanics workshop. This work and the $\mathrm{PhD}$ fundings of T. Pelini and C. Elias were financially supported by the network GaNeX (ANR-11LABX-0014). GaNeX belongs to the publicly funded Investissements d'Avenir program managed by the French ANR agency. R.P. acknowledges support from the Chateaubriand Fellowship Program. 
*e-mail: guillaume.cassabois@umontpellier.fr

[1] A. K. Geim \& I. V. Grigorieva, Nature 499, 419-425 (2013).

[2] K. Watanabe, T. Taniguchi, and H. Kanda, Nat. Mater. 3, 404-409 (2004).

[3] Y. Nanishi, Nat. Photon. 8, 884-886 (2014).

[4] J. Caldwell, I. Aharonovitch, G. Cassabois, J. Edgar, B. Gil, and D. Basov, Nat. Rev. Mater. 4, 552 (2019).

[5] J. S. Lee et al., Science 362, 817-821 (2018).

[6] L. Wang et al., Nature 570, 91-95 (2019).

[7] C. Elias, P. Valvin, T. Pelini, A. Summerfield, C. J. Mellor, T. S. Cheng, L. Eaves, C. T. Foxon, P. H. Beton, S. V. Novikov, B. Gil, and G. Cassabois, Nat. Comm. 10, 2639 (2019).

[8] T.B. Hoffmann, B. Clubine, Y. Zhang, K. Snow, and J.H. Edgar, J. Crystal Growth 393, 1147 (2014).

[9] S. Liu, R. He, X. Z. Du, J. Lin, H. Jiang, B. liu, and J.H. Edgar, Cryst. Growth Design 17, 4932 (2017).

[10] T. T. Tran, K. Bray, M. J. Ford, M. Toth, and I. Aharonovich, Nat. Nano. 11, 37 (2016).

[11] A. Katzir, J. T. Suss, A. Zunger, and A. Halperin, Phys. Rev. B 11, 2370 (1975).

[12] T. Kuzuba, K. Era, T. Ishii, T. Sato, and M. Iwata, PhysicaA\&B 105, 339 (1981).

[13] T. Taniguchi and K. Watanabe, J. Cryst. Growth 303, 525 (2007).

[14] E. Tsushima, T. Tsujimura, and T. Uchino, Appl. Phys. Lett. 113, 031903 (2018).

[15] S. Chichibu, Y.Ishikawa, H.Kominami and K. Hara, J. Appl. Phys. 123, 065104 (2018).

[16] A. Vokhmintsev, I. Weinstein, and D. Zamyatin, J. Lumin. 208, 363 (2019).

[17] A. S. Vokhmintsev, I. A. Weinstein, M. G. Minin, and S. A. Shalyakin, Radiat. Meas. 124, 35 (2019).

[18] C. Attaccalite, M. Bockstedte, A. Marini, A. Rubio, and L. Wirtz, Phys. Rev. B 83, 144115 (2011).

[19] L. Weston, D. Wickramaratne, M. Mackoit, A. Alkauskas, and C. G. Van de Walle, Phys. Rev. B 97, 214104 (2018).

[20] T. Korona and M. Chojecki, Int. J. Quantum Chem. 119, e25925 (2019).

[21] M. Mackoit-Sinkevičienè, M. Maciaszek, C. Van de Walle, and A. Alkauskas, arxiv:1907.02303v1.

[22] P.J. Dean, Prog. Cryst. Growth Charac. 5, 89 (1982).

[23] S. Liu, R. He, L. Xue, J. Li, B. Liu, and J. H. Edgar, Chem. Mat. 30, 6222 (2018).

[24] T.Q.P. Vuong, S. Liu, A. Van der Lee, R. Cuscó, L. Artús, T. Michel, P. Valvin, J.H. Edgar, G. Cassabois, and B. Gil, Nat. Mater. 17, 152 (2018).

[25] T.Q.P. Vuong, G. Cassabois, P. Valvin, A. Ouerghi, Y. Chassagneux, C. Voisin, and B. Gil, Phys. Rev. Lett 117, 097402 (2016).
[26] L.J. Martinez, T. Pelini, V. Waselowski, J.R. Maze, B. Gil, G. Cassabois, and V. Jacques, Phys. Rev. B 94, $121405(\mathrm{R})$ (2016).

[27] G. Grosso, H. Moon, B. Lienhard, S. Ali, D.K. Efetov, M.M. Furchi, P. Jarillo-Herrero, M.J. Ford, I. Aharonovich, and D. Englund, Nat. Comm. 8, 705 (2017).

[28] N. Mendelsohn, Z.Q. Xu, T.T. Tran, M. Kianinia, J. Scott, C. Bradac, I. Aharonovich, and M. Toth, doi:10.1021/acsnano.8b08511.

[29] G. Cassabois, P. Valvin, and B. Gil, Nat. Photon. 10, 262 (2016).

[30] T.Q.P. Vuong, G. Cassabois, P. Valvin, V. Jacques, R. Cusco, L. Artus, and B. Gil, Phys. Rev. B 95, 045207 (2017).

[31] R. Bourrellier, M. Amato, L.H. Galvo Tizei, C. Giorgetti, A. Gloter, M.I. Heggie, K. March, O. Stéphan, L. Reining, M. Kociak, and A. Zobelli, ACS Photon. 1, 857, (2014)

[32] P. Jaffrennou, J. Barjon, T. Schmid, L. Museur, A. Kanaev, J.-S.Lauret, C. Y. Zhi, C. Tang, Y. Bando, D. Golberg, B. Attal-Tretout, F. Ducastelle, and A. Loiseau, Phys. Rev. B 77, 235422 (2008).

[33] G. Cassabois, P. Valvin, and B. Gil, Phys. Rev. B 93, 035207 (2016).

[34] R. Bourrellier, S. Meuret, A. Tararan, O. Stéphan, M. Kociak, L. H. G. Tizei, and A. Zobelli, Nano Lett. 16, 4317 (2016).

[35] M. Cardona and M. L. W. Thewalt, Rev. Mod. Phys. 77, 1173 (2005).

[36] C. Chartrand, L. Bergeron, K. J. Morse, H. Riemann, N. V. Abrosimov, P. Becker, H.-J. Pohl, S. Simmons, and M. L. W. Thewalt, Phys. Rev. B 98, 195201 (2018).

[37] A. Dietrich, K. D. Jahnke, J. M. Binder, T. Teraji, J. Isoya, L. J. Rogers, and F. Jelezko, New J. Phys. 16, 113019 (2014).

[38] H. Akamaru, A. Onodera, T. Endo, and O. Mishima, J. Phys. Chem. Solids 63, 887 (2002).

[39] A. Segura, R. Cuscó, T. Tanigushi, K. Watanabe, G. Cassabois, B. Gil, and L. Artús, to appear in J. Phys. Chem.

[40] K. Koronski, A. Kaminska, N. Zhigadlo, C. Elias, G. Cassabois, and B. Gil, Superlattice. Microst. 131, 1 (2019).

[41] L. Museur, E. Feldbach, and A. Kanaev, Phys. Rev. B 78, 155204 (2008).

[42] M.V. Wolkin, J.Jorne, G. Allan, and C. Delerue, C. Phys. Rev. Lett. 82, 197 (1999).

[43] H. Zhou, H. Alves, D.M. Hofmann, W. Kriegseis, B.K. Meyer, G. Kaczmarczyk, and A. Hoffmann, Appl. Phys. Lett. 80, 210 (2002). 